\title{
Philosophiques
}

\section{Marxisme et condition post-moderne}

\section{Gérard Raulet}

Volume 10, numéro 2, octobre 1983

Le marxisme cent ans après Marx

URI : https://id.erudit.org/iderudit/203231ar

DOI : https://doi.org/10.7202/203231ar

Aller au sommaire du numéro

Éditeur(s)

Société de philosophie du Québec

ISSN

0316-2923 (imprimé)

1492-1391 (numérique)

Découvrir la revue

Citer cet article

Raulet, G. (1983). Marxisme et condition post-moderne. Philosophiques, 10(2), 289-313. https://doi.org/10.7202/203231ar d'utilisation que vous pouvez consulter en ligne.

https://apropos.erudit.org/fr/usagers/politique-dutilisation/ 


\title{
MARXISME ET CONDITION POST-MODERNE
}

\author{
par Gérard Raulet
}

Le marxisme est assurément lié à la modernité. Quand Marx, dès l'Introduction à la Critique de la Philosophie du Droit de Hegel, reproche aux allemands d'être les « contemporains philosophiques du présent ", il invoque l'exemple de la France et de l'Angleterre, à ses yeux les seules «nations modernes".

Certes, la modernité française et anglaise n'a en vérité pour seul mérite que de faire éclater au grand jour les contradictions de la société civile bourgeoise ; mais c'est bien cette réalité sociologique et politique que doit saisir une philosophie authentiquement actuelle.

Toute l'œuvre de Marx, au fur et à mesure qu'elle acquerra ses fondements économiques, sera dès lors dominée par la dénonciation de l'irrationalité du réel - un réel qui est encore loin d'être « à la hauteur " du système hégélien pour le jeune Marx des années 1843-44, un réel livré à l'irrationalité de l'économie capitaliste pour le Marx du Manifeste communiste et du Capital.

On voit ainsi se dessiner, chez Marx lui-même, un rapport complexe entre la rationalité et l'irrationalité, en vertu duquel la modernisation est à la fois l'accomplissement de la Raison et, parce que cet accomplissement reste dominé par la scission (thème hégélien trouvant sa correspondance concrète dans la division de la société en classes), le règne de l'irrationalité.

Que Marx ait voulu supprimer cette irrationalité ne fait aucun doute' ; qu'il s'inscrive par là dans le mouvement d'affirmation de la Raison qui caractérise la modernité semble donc aussi justifier sa répudiation par les tenants actuels de la « postmodernité ». Mais le rapport entre irrationalité et rationalité, la distinction implicite et qui le soustend entre Raison et ratio- 
nalité d'entendement, signalent que la modernité, déjà chez Marx, est en réalité vécue comme une crise de la rationalité incitant à changer le régime dominant du savoir.

La question que posent aujourd'hui les attaques des postmodernes contre le marxisme et leur répudiation globale de la modernité consiste à savoir dans quelle mesure leur propre conscience de vivre un moment de mutation radicale de la société et de la pensée ne trouve pas déjà son archétype dans la conscience que la modernité avait d'elle-même.

Lorsque dans les années 1920-30, Benjamin, Siegfried Kracauer, Ernst Bloch . . . vivent à leur tour le foisonnement culturel de la République de Weimar comme une explosion de la modernité (tout à la fois au sens d'une extraordinaire créativité et d'une auto-destruction), lorsque Walter Benjamin se met, pour cette raison même, à réfléchir, sous la forme d'une " archéologie de la modernité ", au type de rapport à l'histoire qui caractérise la conscience moderne dans la seconde moitié du $\mathrm{XIX}^{\mathrm{ème}}$ siècle, on voit surgir des catégories qui modifient profondément le marxisme et qui, par ailleurs, peuvent encore nous servir aujourd'hui pour appréhender la « crise post-moderne ».

Il convient donc de se demander si la post-modernité sonne véritablement le glas du marxisme comme philosophie de l'histoire ou si elle ne représente pas plutôt une nouvelle crise de la modernité, incitant il est vrai à repenser radicalement le rapport entre la théorie et la pratique.

\section{MODERNITÉ ET MODERNISATION : DIALECTIQUE DE LA RAISON}

Quant aux signes distinctifs de la modernité comme société industrielle dominée par la primauté de la technique et quant aux caractéristiques majeures de la société post-industrielle, marquée par la primauté du savoir, tous sont au fond d'accord. C'est à propos de la signification de cette mutation - crise dialectique ou rupture radicale - que les opinions divergent. Comparées aux sociétés traditionnelles, pré-industrielles, que caractérise la cohésion organique du monde vécu, les sociétés modernes industrielles trahissent certes l'émergence de scissions, avec d'une part la différenciation de sphères autonomes et d'autre part la polarisation de l'antagonisme de classes, mais elles « tien- 
nent " encore par la vertu d'un concept de vérité se réalisant dans l'histoire qui porte d'abord l'affirmation de la classe bourgeoise, puis la conception de l'émancipation du prolétariat. Il faudra attendre les années 1930/1970 pour que cette idéologie du progrès batte de l'aile sous les coups successifs de ses effets négatifs sur le mouvement ouvrier allemand (cf. Benjamin, Thèses sur la philosophie de l'histoire), de la décomposition du mouvement ouvrier occidental, de la nouvelle étape de la modernisation sociale que signale cette décomposition ${ }^{1}$, de l'évolution du marxisme soviétique et, au niveau beaucoup plus profond de l'alternative ontologique du Tout et du Néant, des deux traumatismes du Mal radical (Auschwitz) ${ }^{2}$, et d'une possible destruction cosmique par le progrès technique ${ }^{3}$. Quand l'idéologie du progrès s'effondre, il faut abandonner ce que Lyotard appelle le « Grand Récit » de la Raison dans l'Histoire et de l'émancipation. Cette crise dite "post-moderne » est essentiellement une crise de la continuité, fût-elle dialectique.

Notre but n'est pas ici de reconstituer les causalités directes ou indirectes, et notamment économiques et sociales, qui déterminent ce moment critique mais plutôt de considérer l'irruption du discontinu et de la scission dans la continuité de la modernité comme une situation typique qui s'est plusieurs fois répétée dans l'histoire - et en particulier sous Weimar - et à partir de laquelle il peut être possible d'interroger l'époque présente. Celleci se définit au demeurant en fonction de la modernité, dont elle tire le bilan, que ce soit en répudiant une continuité qui y a manifesté sa faillite et qu'il serait a fortiori vain de prôner encore dans le contexte de la société post-industrielle, ou bien encore en y voyant au premier chef, comme Daniel Bell, l'aggravation ininterrompue d'une scission qui est devenue irréversible et qui fait donc là aussi partie intégrante des conditions régissant l'existence de l'âge moderne. Faut-il se résoudre au relativisme et à l'éclectisme (dont l'architecture post-moderne offre un exemple érigé en programme), faut-il prôner, comme le fait Lyotard dans La condition post-moderne, le caractère absolument agonistique des jeux de langage, faut-il admettre, comme

1. HORKHEIMER, Théorie traditionnelle et théorie critique, Gallimard, 1974.

2. C. BLOCH et ADORNO.

3. Cf. BLOCH, Experimentum mundi, traduction française par Gérard RAULET, Payot, 1981. 
le fait Daniel Bell, que la différenciation du monde vécu traditionnel en sphères autonomes (science, droit et morale, art) dans laquelle Max Weber (et après lui Habermas) voit l'essence de la modernité, débouche sur une « disjonction toujours plus profonde de la structure sociale (économie, technologie, organisation socio-professionnelle) et de la culture (expression symbolique des significations) plongeant les sociétés occidentales dans un état de crise permanente » et rendant irrévocablement anachronique une conception de l'histoire et de l'émancipation qui recomposerait dialectiquement ces sphères dissociées ? ${ }^{4}$. Dans tous les cas.il s'impose d'envisager la modernité sous le double éclairage de la continuité et de la scission. Si l'on veut s'acheminer ici vers un examen critique de la rupture avec le modèle de la vérité dans l'histoire, si l'on veut s'expliquer également comment Habermas lui-même, dans son dernier ouvrage, Théorie des kommunikativen Handelns ${ }^{5}$, peut tout à la fois entériner l'idée d'une coupure radicale mettant fin à l'épistémè moderne et dénoncer l'abandon du "projet inachevé de la modernité ", désigner le concept hégélien de vérité comme un "déficit " de la Théorie critique et pourtant viser une synthèse qui recomposerait ce qui fut dissocié, il semble utile de reconstruire d'abord la « dialectique de la modernité » qui s'accomplit parallèlement à la dialectique de la Raison décrite par Horkheimer et Adorno en 1944 et d'y déterminer le statut de l'expérience de la scission comme un moment dialectique précisément occulté par l'idéologie de la continuité ou en passe d'être absolutisé par les tenants d'une coupure irrémédiable.

La continuité qui porte l'accomplissement de la modernité se fonde en fait sur la réalisation impérialiste de la rationalité d'entendement. Cette constatation qui passe aujourd'hui pour un lieu commun ne s'imposa aux esprits, bien qu'elle eût été expressément développée par la théorie wébérienne de la rationalisation, que dans les années 20-30. On la trouve alors dans les écrits de droite et de gauche, rationalistes ou irrationalistes,

4. LyOTARD, La condition post-moderne, Minuit, 1979 ; D. BELL, Vers la société post-industrielle, Laffont, 1976.

5. HABERmas, Théorie des kommunikativen Handelns (désigné infra par les initiales TKV), Frankfurt, 1981 : cf. le dernier chapitre ; voir aussi l'entretien "Dialektik der Rationalisierung ", in Aesthetik und Kommunikation, octobre 1981. 
conservateurs ou progressistes : Jaspers, Heideberg, Spengler, Moeller van den Bruck, puis les membres de l'Institut de Recherches sociales de Francfort. Le dénominateur commun de ces courants en apparence inconciliables est qu'ils réagissent tous aux effets de la modernisation et au phénomène de la modernité. Cette expérience partagée et les échanges théoriques qu'elle motive attirent l'attention sur la circulation des concepts, sur les emprunts et les héritages, et incitent à la prudence à l'égard de toute schématisation polémique (rationalisme contre irrationalisme, modernité contre post-modernité — ou l'inverse).

La Dialectique de la Raison d'Adorno et Horkheimer ainsi que la Critique de la Raison instrumentale publiée par ce dernier sous le titre Éclipse de la Raison (la simple comparaison de ces deux appellations - dialectique et éclipse - signalant à elle seule la problématique) permettent de comprendre que la modernisation a en réalité fait triompher la rationalité d'entendement de la science et de la technique, soit un aspect seulement de la Raison du XVIIIème siècle : "Si par Lumières et progrès intellectuel nous voulons dire libérer l'homme de la croyance superstitieuse en des forces mauvaises, aux démons et aux fées, au destin aveugle, bref l'émanciper de toute peur, alors la dénonciation de ce qui est communément appelé raison est le plus grand service que la raison puisse rendre ${ }^{6}$. La Raison part en guerre contre la Raison ! Elle se dénonce elle-même, pour autant qu'elle puisse le faire - ce qui reste à montrer - en prenant conscience qu'elle s'est elle-même disqualifiée : " (Ce livre) se propose d'examiner le concept de rationalité sous-jacent à notre culture industrielle contemporaine, afin de découvrir si ce concept ne renferme pas des défauts qui en altèrent l'essence même " ${ }^{7}$. Comme la Dialectique de la Raison rédigée en collaboration avec Adorno, l'ouvrage de Horkheimer n'attaque en fait pas seulement une époque déterminée de la Raison, mais une " maladie inséparable de la nature de la Raison ", depuis le temps où elle naquit « de la tendance impulsive de l'homme à dominer la nature ${ }^{8}$. Toutefois si la maladie de la raison est en quelque sorte congénitale, c'est bien l'industrialisme (ibid.) qui, tout à

6. HORHKEIMER, Éclipse de la Raison, traduction française de J DEBOUZY, Payot, 1974 : p. 193.

7. Ibid., p. 9.

8. Ibid., p. 182. 
la fois, en a manifesté la gravité et a placé la civilisation dans cette situation véritablement critique où se décidera l'alternative d'une auto-destruction et d'une éclipse de la Raison ou d'une transformation et d'une émancipation.

En même temps, le langage et l'art apparaissent comme les points nodaux d'une transformation interne de la rationalité : le langage se révèle être un instrument de manipulation d'une efficacité terrifiante9, mais aussi le témoin du singulier, perpétuant une expérience mimétique anéantie par la formalisation et les possibilités qu'elle crée : rendre toutes choses manipulables ou échangeables ; l'art « donne à la nature l'occasion de se refléter dans le royaume de l'esprit »(ibid.), il la récrée dans son langage plutôt qu'il la domine ou la manipule ${ }^{10}$. Si la Raison peut être sauvée, c'est par une philosophie dont le langage fonctionnerait comme celui de l'art. Ce thème de l'expérience esthétique et d'un devenir-esthétique de la philosophie reviendra ici avec insistance.

\section{L'ART ET L'EXPÉRIENCE DE LA SCISSION}

Au point où nous en sommes, cette importance reconnue à l'art invite à reconstituer son destin, depuis le moment où les Lumières ont identifié la connaissance au modèle de la physique. De ce destin se dégage une dialectique qui détermine tout à la fois pourquoi l'art et ce qui s'est accompli comme raison sont devenus étrangers l'un à l'autre, se sont dissociés, et pourquoi l'art s'est vu investi d'un potentiel de protestation.

Parallèlement à l'autonomisation de la rationalité d'entendement qu'accomplit la modernisation se sont affirmés une morale et un art autonomes qui chez Kant, dans la perspective régulatrice comme dans la conception téléologique de l'histoire, n'auraient pas dû être dissociés mais au contraire s'unir à la connaissance d'entendement au fur et à mesure des progrès de la rationalisation. Détruisant les traditions par la modernisation sans pouvoir cependant se passer du potentiel de motivation et

9. Jeu de discours stratégique, comme le dira plus tard haBERMAS (voir dans Éclipse de la Raison : p. 183), ou système de signes qui « doit déchoir en stratégie " (Dialectique de la Raison, traduction française de E. KAUFHOLZ, Gallimard, 1974 : p. 35).

10. La Dialectıque de la Raison est plus précise et insiste sur la recréation ; l'art devient sinon positivisme ; voir p. 35. 
de légitimation qu'elles représentaient, le monde bourgeois trouve dans la morale privée et dans l'art autonome les valeurs qui lui feraient sinon défaut. Selon la démonstration aujourd'hui classique de Max Weber, l'éthique protestante devient l'esprit du capitalisme. "Mais les bases de ce bel édifice seront bientôt sapées - par le capitalisme lui-même. L'éthique protestante ne résistera pas à la production de masse et à la consommation de masse, aux efforts sans cesse renouvelés pour implanter un mode de vie fondé sur la recherche du confort et du plaisir ${ }^{11}$. La difficile mais indispensable adéquation entre une codification formelle et universalisable et l'intériorisation des normes ${ }^{12}$ échoue et se décompose de plus en plus. Dans l'affirmation du moi autonome, l'art va jouer un rôle décisif.

L'art autonome, mis sur le banc des accusés par la sociologie post-moderne, n'a - précisément parce qu'il était autonome joué qu'un temps le jeu de la sublimation compensatrice. D'emblée investi, chez Kant, du pouvoir de symboliser une harmonie que le réel refuse encore, il représente un potentiel de révolte. Dans l'esthétique « affirmative » (Marcuse) du classicisme, cette protestation se cristallise mais s'épuise, se neutralise aussi dans la transparence de la belle apparence. Or, si l'art autonome est ainsi le domaine dans lequel s'est réfugiée l'harmonie, il devient du même coup inévitablement le terrain sur lequel la scission va se faire entendre ; il conserve de la belle apparence l'idée d'un monde impossible, une "promesse de bonheur ${ }^{13}$ inaccessible qui le coupe de plus en plus du monde et le retranche dans son autonomie (esthétisme et art pour l'art) ${ }^{14}$. Lorsque Baudelaire intervient, l'échec de la Révolution de 1848 - comme jadis pour Schiller le passif de la révolution française : la tyrannie et la terreur — ${ }^{15}$ le conduit à penser esthétiquement le monde moderne mais sans espoir de le changer et seulement pour en traduire esthétiquement l'irréconciliation : «Le poète accepte et pourtant n'accepte pas le déchirement et la scission qu'il constate au sein du réel, entre le réel et lui-même, entre le réel

\footnotetext{
11. BELL, op. cit., p. 413.

12. HABERmas, Raison et légztımité (désigné infra par RL), op. cit., p. 121 sq, et p. 129.

13. habermas, « La modernité, un projet inachevé ", Critique, octobre 1981 : p. 961.

14. Ibid., et RL, p. 120.

15. Notions que nous retrouverons dans la discussion de la post-modernité.
} 
et l'idéal ou le possible ${ }^{16}$. Depuis les fragments sur Baudelaire de Walter Benjamin, " nous ne voyons plus Les Fleurs du Mal comme un repliement de la poésie sur elle-même sous le signe exclusif de l'art pour l'art, mais comme le témoignage d'une expérience historique qui a su faire accéder à la transparence de l'art les transformations de la société du XIX ${ }^{\text {ème }}$ siècle $"{ }^{17}$. Schiller voulait faire de la politique une œuvre d'art, réaliser la belle harmonie ; après lui, le romantisme apparaît avant tout comme l'expression désespérée de l'impossibilité d'une telle réalisation et cette expérience ne va cesser de s'aggraver au cours du XIX ${ }^{\mathrm{ème}}$ siècle, engendrant d'abord l'art pour l'art, puis l'avant-garde. Il faudrait être la Käthchen von Heilbronn de Kleist et jouir tout à la fois de la grâce divine (Gnade) et de la grâce schillerienne (Anmut) pour échapper à l'expérience de la scission. Seul le postulat de l'agencement merveilleux du monde, dans lequel les hommes sont faibles mais perfectibles (le Comte de Strahl ou l'Empereur dans la pièce de Kleist) permet à la naïveté de triompher ; mais cette possibilité n'est préservée que par la communication arbitraire entre la grâce divine et celle de l'individu ainsi que par le maintien irréel ${ }^{18}$ d'un ordre médiéval irrémédiablement révolu et dont Kleist sait fort bien qu'il ne peut être restauré ${ }^{19}$. Si l'on suspend ce postulat, cette communication «dialectique » (au sens kantien) et cet anachronisme volontairement utopique, c'est au-delà du monde qu'il faut chercher un ordre nouveau (Pentbésilée). Il faut transgresser le désordre dans l'horrible pour atteindre au-delà du monstrueux, conçu comme expiation purificatrice (le meurtre du Grec Achille), le sublime d'une nouvelle Fondation (Penthésilée accomplit jusqu'en ses conséquences les plus extrêmes la contradiction de l'amour et de la mort qui régit l'État des Amazones et, par sa propre mort, le délivre de sa malédiction). Monstrueux et sublime sont en continuité : ils vont tous deux au-delà de la belle apparence qui masque le désordre de l'histoire (l'illusion de l'amour dans les scènes 14 et 15). Si la beauté et la grâce sont toutes de

16. H LEFEBVRE, Introductıon à la modernité, Minuit, $1962:$ p. 174 sq.

17. H R JAuss, Pour une esthétique de la réception, traduction française de C. MAILLARD, Gallimard, 1978 : p. 201.

18. Les interprètes ont souvent souligné l'irréalité du Vèrne acte.

19. Acte 1, scène 1 : Catherine doit quitter le monde clos de la ville ceinturée de murs et de la famille bourgeoise pour se lancer sur les chemins de l'histoire (l'historicité est symbolisée par le rythme échevelé du deuxième acte, inspiré des romans de chevalerie). 
mesures, mais sont illusion ou - dans le meilleur des cas exception individuelle (Käthchen) et à ce titre désespérée, le salut est dans la démesure. L'apollinien doit céder la place au dionysiaque, au déchaînement des forces vitales. Dans la Penthésilée de Kleist sont anticipées, par la destruction de la mesure grecque, les catégories au moyen desquelles Nietzsche rejettera l'hellénisme de Winckelmann et le dénoncera comme une illusion moderne. Le tragique kleistien illustre parfaitement ce que dit Hegel de l'art romantique comme décomposition de l'interpénétration de l'esprit et du sensible qui caractérisait l'art classique $^{20}$; il est aussi protestation contre la modernité, bien avant que Nietzsche n'en entreprenne la critique, et déjà au moyen de catégories qui anticipent l'opposition nietzschéenne de la démesure vitale à la Raison.

Nous nous sommes attardés sur Schiller et sur Kleist, d'une part parce que les catégories dégagées au passage (tyrannie, barbarie, terreur, monstrueux, sublime) traduisent l'auto-destruction de la Raison, d'autre part parce que l'expérience de la scission qui est celle de la modernité semble bel et bien produire ce nietzschéisme que Habermas stigmatise chez les postmodernes. Si tel est le cas, la post-modernité entre dans le schéma typique des crises de la modernité.

\section{DU MODERNE AU POST-MODERNE}

Derrière l'irrationalité apparente, voire l'anti-rationalisme déclaré de la scission, se cache donc une dialectique de la modernité coextensive de la dialectique de la Raison. Conçues ainsi, la continuité et la rupture procèdent d'un même mouvement ; le geste moderne, mais aussi la vanité de sa révolte sont inscrits en lui. On peut dès lors, comme le fait Habermas dans " La modernité, projet inachevé », à la fois constater la faillite de la modernité et la réhabiliter en la réinscrivant dans la continuité historique. Pour pouvoir être écrite, l'bistoire de la modernité doit cependant privilégier en quelque sorte ce moment de rupture, car c'est seulement à partir de lui que la perversion d'une continuité qui se révèle être une trahison devient perceptible. La réhabilitation de l'échec

20. Cf. sur ce point P BURGER, Théorie der Avantgarde, Suhrkamp, 1974 : p. 128. 
révèle la dialectique. L'histoire de la Raison semble avoir détruit la Raison dans l'histoire; mais la rupture du continuum, y compris sous les formes irrationalistes qu'elle affecte, signale que cette raison dans l'histoire avait pris la forme du mauvais infini de la rationalité d'entendement et que pour cette raison même sa continuité s'était établie sur la base d'une scission irrévocable dont le geste esthétique de rupture, procédant de l'autonomie que cette scission avait constituée, témoigne. Par là même, il témoigne tout autant contre la «Raison » que pour une transformation de la rationalité. Il n'est dès lors plus aussi certain qu'il faille renoncer à la Raison et à l'bistoire, même s'il n'est pas sûr non plus que cette transformation de la rationalité puisse encore s'investir dans une dialectique bistorique accomplissant la Raison.

Nous retrouvons l'inévitable question des agents d'une telle transformation; or, dès les années trente, la Théorie critique fait l'expérience que le modèle lukacsien est devenu intenable. Quatre modèles au moins se font face, sur lesquels nous reviendrons : celui de la "Grande Rupture " avec le Logos disqualifié, la "barbarie positive " de Benjamin (positives Barbarentum), selon laquelle la réification extrême crée elle-même les conditions d'un renversement révolutionnaire de la culture dominante, la dialectique négative qui, partant des mêmes prémisses - réification totale - maintient à tout le moins l'auto-réflexion d'une philosophie témoignant de la réification, et enfin le projet habermasien d'une rationalité communicationnelle. Dans la dialectique négative d'Adorno, le moment de rupture n'est possible que comme auto-réflexion d'une implication insurmontable : "La liberté de la philosophie n'est rien d'autre que le pouvoir de faire entendre sa non-liberté ${ }^{21}$. La philosophie, en " faisant entendre », répète de façon quasi-esthétique l'impasse de l'histoire et ne sauve la Raison que par la vertu qu'elle lui conserve de témoigner de la trahison de ses promesses. Elle répète la révolte et l'échec nécessaires par lesquels l'art faisait entendre la scission.

Ce mouvement de la pensée préserve à tout le moins l'autoréflexion. Et il le fait surtout de façon telle que la rupture n'est re-saisie que par cette auto-réflexion. En sorte que même dans ce modèle extrême la rupture est réfléchie comme moment dia-

21. Negattve Dralektik, Suhrkamp, 1966 : p. 27. 
lectique. Elle permet alors de briser de l'intérieur la réification totale engendrée par le destin de la Raison. Mais, à l'inverse, cette négation ne peut s'accomplir qu'en pensant le système de la réification et de l'administration totales. Si le monde administré tend vers le système, la philosophie doit "garder du respect pour le système ${ }^{21 \text { bis }}$. La possibilité de la pensée se constitue dans le redoublement réfléchi de son impuissance, dans la reconstruction du mouvement qui a scellé sa faillite. Le mouvement de la dialectique négative ne fait certes que penser la réification ; mais, par là même, elle pense l'impensé. Dans la civilisation technicienne du monde administré, la pseudo-cö̈ncidence du sujet et de l'objet n'est jamais qu'une soumission du premier au second - soumission rendue impensable par l'illusion qui habite le sujet de dominer et de maîtriser l'objet. Si l'identité est devenue réification, le concept qui pense l'identité s'abolit. La soumission de l'objet au sujet se transforme irréversiblement en soumission du sujet à la réification. L'objet est ce qui ne peut plus être pensé par un sujet qui n'existe plus puisqu'il s'est résorbé en lui. En se rendant à nouveau possible par le redoublement de son impossibilité, la pensée pense à nouveau en pensant l'objet. Le moment subjectif et l'objectif redeviennent différents. Telle est la possibilité que recouvre la notion de constellation, « communication de ce qui est différent ». Synthèse ponctuelle et révocable qui est moins une identification qu'un accord dans la différence - non point :malgré la différence et sur le mode du compromis, ou de l'assimilation et de la réduction, mais au sein de la différence, par elle - la constellation sauve à la fois le moment subjectif et l'objet ; elle préserve le singulier et le particulier au sein même de leur rencontre. C'est en ce sens qu'elle se réclame de l'esthétique, de cette synthèse anticipée que définit l'expérience esthétique kantienne en voyant dans le Beau le symbole de l'idée, de l'universel, réalisé dans un objet particulier.

Résumons : la rupture témoigne d'une continuité - une continuité dialectique qui accomplit elle-même la scission; si elle a un sens, c'est en fonction de 'cette dialectique. Dans le modèle adornien, développé ici parce qu'il est le plus extrême 
et en apparence le plus désespéré, la continuité aboutit à une impasse qui clôt l'ouverture de l'histoire ; la Raison ne peut plus être réalisée dans l'Histoire, parce qu'elle s'est déjà réalisée sous une forme totalitaire. Mais penser cette clôture ouvre en elle une brèche qui assigne à nouveau un statut dialectique à la rupture. Précisément parce qu'elle est prise au sérieux comme l'effet d'un mouvement dialectique, elle peut être pensée dans une dialectique. La prendre au sérieux n'implique nullement le verdict d'une Grande Rupture, l'impasse de la Raison dans l'Histoire n'implique nullement le renoncement à la dialectique, ni à la raison qui se pense encore, ni peut-être même à l'histoire si le mouvement d'intégration dialectique de la rupture y est pris en charge par des forces réelles, mais on ne peut plus simplement affirmer que la Raison et la révolution sont en marche.

\section{L'INSTANT ET L'HISTOIRE, LE NOUVEAU ET LA DURÉE}

La XI ${ }^{\text {ème }}$ thèse sur la philosophie de l'histoire de Walter Benjamin souligne que la foi dans un "sens de l'histoire " fut fatale au marxisme des années 1891 (programme d'Erfurt) à 1933 (effondrement de la République) : «Rien ne fut plus corrupteur pour le mouvement ouvrier allemand que la conviction de nager dans le sens du courant ». Et Benjamin ne se fait pas faute de rappeler que ce courant-là fut celui d'une certaine rationalité : celle du développement technique. Il faut donc, contre l'historisme - qu'il nomme aussi conformisme - rébabiliter dans le marxisme le moment de la rupture. Faute de quoi (thèse VIII) le fascisme nous apparaît comme une " surprise ». L'impossibilité de penser la rupture est propre à l'idée d'un " progrès continu de l'espèce humaine . . . dans un temps homogène et vide " (XIII). Le renouvellement dialectique du marxisme dans les années vingt procède, chez Benjamin comme chez Bloch, du refus de ce temps homogène. Benjamin semble avant tout opposer au temps homogène et vide le moment de la rupture, un Kairos qu'il nomme aussi "moment moderne "; cependant, pour que cette rupture ne soit pas elle-même vide, il la dote d'un contenu qu'elle puise dans un rapport particulier au passé. Il se réfere à la mode, qui prend soudain conscience de ce qu'il y a d'à-présent dans tel ou tel moment de l'histoire, s'en inspire et l'actualise : c'est cette actualisation brutale, tranchant dans le 
continuum historique, qui constitue la rupture. Ce rapport en apparence sans loi obéit toutefois à une logique : celle du présent au moment même où il se tourne vers le passé, et c'est précisément cette logique, qui nous paraît irrationnelle, qu'il s'agit de saisir. La modernité est l'état historique dans lequel le rapport à l'histoire ne peut plus adopter la forme de la continuité homogène, car l'histoire de la modernité est celle d'une scission ; elle se définit comme le moment où le flux de l'histoire, en s'identifiant à un fragment du passé qui prend brutalement un sens, s'interrompt et signale un moment de décision qui cherche à se penser lui-même et en tous cas une rupture qui ne peut plus s'identifier à la continuité qui la porte : « devenir mâtre d'un souvenir tel qu'il brille à l'instant d'un péril " (Thèse VI). Tout comme aux heures révolutionnaires les hommes empruntent leurs costumes aux grands modèles (18 Brumaire), la posture nietzschéenne de la post-modernité pourrait être en ce sens une mode. La rupture est une "illumination profane ». Pour comprendre le sens exact de ce rapport au passé qui est réception et héritage (au sens blochien) mais sur un mode qui, bien plus que chez Bloch, privilégie l'inattendu, on peut se référer à une mise en garde d'Adorno. Adorno écrit dans la Théorie esthétique : "Rien n'est plus préjudiciable à la connaissance théorique de l'art moderne que sa réduction à des ressemblances avec l'art antérieur. Par le principe du 'Tout a déjà étés sa spécificité s'évanouit. On le nivelle précisément sur le continuum non dialectique et sans failles d'un développement monotone qu'il fait éclater . . . L'immersion dans la dimension historique devrait permettre de résoudre ce qui, jadis, resta insoluble. C'est uniquement dans cette perspective qu'il convient de rattacher le présent au passé ${ }^{22}$. Quelque chose qui n'a pas de nom s'identifie à la fois péremptoirement et fugitivement à un fragment du passé ; mais c'est par lui que ce passé prend sens et non l'inverse : c'est là le point de vue de la post-histoire. Le moment de la rupture sort de l'histoire - et c'est de la même façon qu'il " sort par effraction du cours de l'histoire une époque déterminée " (Thèse XVII). En ce sens précisément, la post-modernité rompant avec l'bistoire pourrait bien être l'expérience renouvelée de la modernité. Le modèle de la dialectique négative développé plus haut suggère la même

22. ADORNO, Théorie esthétique, traduction de M. JMENEZ, Klincksieck, 1974 : p. 33. 
notion de post-histoire : c'est en redéployant une histoire qui s'est close et donc en adoptant le point de vue post-historique de son achèvement - du moins potentiel - que le présent cherche à sortir de l'enfermement auquel l'a condamné la dialectique de la Raison. L'illumination est l'instant de rupture où, dans le continu ou au sein de cette clôture, le nouveau fait irruption : "Effectué en plein air, le même saut est le saut dialectique, la révolution" (Thèse XIV). Alors seulement le "sens de l'Histoire » peut être tranché - tout à la fois contre ceux qui le connaîtraient à l'avance et contre ceux qui prétendraient qu'elle n'en a plus ; l'instant illumine le passé qui « par une secrète sorte d'héliotropisme tend à se tourner vers le soleil en train de se lever dans le ciel de l'histoire " (Thèse IV) ${ }^{23}$.

\section{ÉCLECTICISME ET HISTORISME}

Le rapport au passé qui caractérise l'architecture postmoderne se distingue radicalement de cet héliotropisme en refusant précisément toute centration, fût-ce sur un nouveau encore en train de se former. Le présent n'est plus tournant, "fusion du passé et de l'avenir dans le présent brûlant » (Jünger), tension messianique entre la commémoration et l'attente du nouveau comme chez Benjamin. Pour les post-modernes, la rupture signalée par la modernité esthétique et son échec ouvre une ère postérieure à la rupture, une ère de décomposition du sens qui impose un éclectisme radical (Jencks). Dans un monde de flux non liés, déterritorialisés, où l'ordre du discours se manifeste comme agonistique généralisée (Lyotard) ${ }^{24}$, l'instant situé au carrefour des relations pragmatiques déterminant la rencontre des actes de langage est simplement l'occasion, le Kairos d'une invention : «On peut faire un coup pour le plaisir d'inventer » (ibid.). Cette conception semble s'opposer au refus, par le post-modernisme architectural, de "l'innovation incessante et (de) la révolution continuelle ${ }^{25}$ - refus au nom duquel l'architecture post-moderne conçoit son rapport au passé sur le mode de l'historisme. Il n'en est rien, pour deux raisons essentielles : la disparition des codes

23. La traduction des Thèses est de M. de Gandillac, in L'Homme, le langage et la culture, Denoël, 1971 ; c'est nous qui soulignons.

24. LYOTARD, La condition post-moderne, Minuit, $1979:$ p. 23.

25. Charles JENCKs, The language of post-modern architecture, London Academy, 1977 : p. 6. 
susceptibles d'introduire un ordre à la fois dans la référence au passé et dans les jeux $d u$ discours présents ainsi que la nature du lien social présupposé dans l'un et l'autre cas.

En s'accomplissant, la Raison est disqualifiée - thèse qui permet une appropriation post-moderne de la Dialectique de la Raison d'Adorno et Horkheimer. Mais puisqu'aussi bien elle s'est réalisée, sa disqualification signifie son abolition dans les faits. Dans une ère de décomposition réelle dont l'agonistique serait la véritable logique, le mode de production n'est plus même celui de la réification mais celui de la délocalisation du désir ${ }^{26}$. Dès lors le post-modernisme s'installe dans la rupture. Les postmodernes se contentent de reprendre l'idée de synthèse sous la forme non dialectique d'une juxtaposition des codes, notamment ceux de la lecture commune et de la lecture des experts. La notion de multiple codage proposée par Charles Jencks doit permettre à l'architecture de s'adresser à tous, aux larges masses comme à l'élite ; c'est elle qui justifie l'éclectisme du style et le recours arbitraire aux styles passés, c'est-à-dire l'historisme post-moderne. Du même coup, les post-modernes prennent leurs aises dans la décomposition de la modernité et ne cherchent plus à faire de la rupture qu'elle représente un tournant. Le post-modernisme, du moins dans sa version architecturale, représente une forme de positivisme en ce sens qu'il repose sur l'acceptation d'une rationalité technicienne qu'on renonce à critiquer. Le positivisme consiste à constater la décomposition et à la refléter : le multiple codage est au fond ce que Deleuze appelle le "décodage ", la destruction des codes, la " déterritorialisation " accomplie par le capitalisme.

Le post-moderne "flaire dans la brousse de l'autrefois le fumet de l'actuel »(Benjamin), mais uniquement pour alimenter un éclectisme radical dont la nature composite reflète la décomposition. Il admet ce que Lyotard décrit comme le « lien social post-moderne ": "l'atomisation du social en souples réseaux de jeux de langage " rendant la conception moderne de consensus anachronique. Mais il y a là un paradoxe : par ses recours historistes à l'histoire, l'éclectisme s'assure au niveau

26. Cf. DeleuZe-guattari, Capitalisme et schizophrénie, tome I : L'Anti-Oedipe, Minuit, 1972 ; lyotard, Économie libidinale, Minuit, 1974 ; Marc Gulllaume, Le capital et son double, P.U.F., 1975. 
purement formel la légitimité d'une tradition qu'il se refuse certes à invoquer comme telle mais qui, en liaison avec la pluralité des lectures possibles, semble vouloir constituer le consensus le plus large possible, appuyé sur un public d'une extrême diversité. La nostalgie du consensus n'a pas disparu. L'attitude de Lyotard est plus radicale : il ne conçoit plus ce consensus que comme un contrat temporaire et purement local ${ }^{27}$, conformément à l'évolution constatable des interactions sociales. L'architecture post-moderne propose quant à elle une participation des citoyens qui, ne passant plus par les canaux officiels, rejoint tout à fait cette notion de contrat localisé. En sorte que dans une version pluraliste rejoignant le libéralisme, l'éclectisme, l'historisme, l'agonistique, etc., se présentent comme l'acceptation d'une seconde (ou troisième) nature née de l'échec de la Raison et que la Raison ne peut donc plus prétendre mâtriser.

De façon tout à fait surprenante, la pensée post-industrielle ou post-moderne rejoint ici, sinon chez Daniel Bell, la croyance en une autostabilisation qui avait été celle du libéralisme bourgeois, puis de la glorification de la pensée technicienne, puis enfin de la Systemtheorie et du fonctionnalisme des théoriciens du système (auxquels Lyotard s'oppose pourtant vivement) ${ }^{28}$. Elle n'est point tant, comme le dit Habermas, une volonté de régresser vers des formes de vie "indifférenciées ", c'est-à-dire vers la cohérence organique d'un monde vécu prémoderne ${ }^{29}$, mais plutôt l'acceptation d'une complexité insurmontable qu'il convient de laisser jouer. Et il est vrai d'ailleurs que les tentatives pour la gérer confirment sans cesse l'impuissance de la raison. De cette raison qui ne cesse d'échouer, on fait simplement abstraction ; on renonce à en entreprendre la critique pour admettre que sa disqualification crée les conditions de son abolition de facto.

Le libre jeu des actes de langage est censé garantir la plus grande démocratie ; mais du fait même que la rationalité tech-

27. LYOTARD, La condition post-moderne, op. cit., p. 107.

28. Cf. HABERMAS, RL, chap. 5, et Théorie et pratique, tome 2 : "Conséquences du progrès scientifique et technique ", Payot, 1975 ; position de Lyotard à l'égard de la Systemtheorie, op. cit., p. 25.

29. "Moderne und post-moderne Architektur ", in Die Andere Tradition Callwey, 1981 ; traduction française de G RAULET, in catalogue du Festival d'Automne de Paris, Éd. du Moniteur, 1982. 
nicienne et technocratique subsiste, le jeu de discours stratégique ne laisse de menacer ce vœu pieux. Lyotard doit symptomatiquement le reconnaître à la fin de La condition post-moderne en laissant ouverte une alternative: "L'informatisation des sociétés ... peut devenir l'instrument 'rêvé» de contrôle et de régulation du système du marché, étendu jusqu'au savoir luimême, et exclusivement régi par le principe de performativité. Elle comporte alors inévitablement la terreur. Elle peut aussi servir les groupes de discussion sur les métaprescriptifs en leur donnant les informations dont ils manquent le plus souvent pour décider en connaissance de cause. La ligne à suivre est simple en principe : c'est que le public ait accès librement aux mémoires et aux banques de données ${ }^{30}$. Pétition de principe, wishful thinking.

\section{DÉCOMPOSITION E'T STRATÉGIE DIALECTIQUE}

Contre l'acceptation de la décomposition, une approche dialectique requerrait qu'on dialectise l'alternative de la démocratie et de la terreur. Les développements précédents auront cependant permis de comprendre que cette dialectisation doit procéder de l'intérieur de la rupture, parce que celle-ci ne peut être niée mais est au contraire elle-même une réalité dialectique engendrée par la dialectique de la modernité. Entreprise au sein de la décomposition, cette démarche implique alors le respect des discontinuités et du singulier.

Le post-modernisme érige en vision du monde l'incapacité à dialectiser, dans un mouvement d'auto-réflexion rétablissant la communication des sphères dissociées, les jeux de discours fondamentalement différents de l'art (l'expressif), de la morale (le normatif) et de la technique (la performativité). Il y a plus : l'acceptation de leur dissociation retombe dans le temps cyclique. La modernité marqua la rupture définitive avec les modèles cycliques $^{31}$, le renoncement à la modernité semble les rétablir. C'est le cas si l'on cesse de comprendre la mode dans l'optique des Thèses sur la philosophie de l'bistoire de Benjamin (cf. supra)

30. LYOTARD, op. cit., p. $107 \mathrm{sq}$.

31. Cf. JAUSs, "La modernité dans la tradition littéraire ", in Pour une esthétique de la réception, op. cit. 
pour en faire un phénomène « où resurgissent toutes les formes du passé (archaïques, folkloriques, rustiques, traditionnelles) vidées de leur substance mais exaltées comme signes dans un code où tradition et néo, ancien et moderne s'équivalent et jouent alternativement ${ }^{32}$. La post-modernité architecturale adopte précisément cette attitude. On en trouve également la trace dans cette idée de Lyotard : "Une œuvre ne peut devenir moderne que si elle est d'abord post-moderne. Le post-modernisme ainsi entendu n'est pas le modernisme à sa fin, mais à l'état naissant et cet état est récurrent ${ }^{33}$. Il s'agit certes ici de souligner que toute manifestation esthétique moderne intervient comme la sanction d'une modernité (celle que nous avons développée plus haut comme histoire dialectique de la modernité) et qu'elle est à ce titre, en tant que rupture, un moment post-historique. Mais si l'on généralise ainsi (récurrence) cette post-historicité constitutive, modernité et post-modernité se succèdent de façon mécanique (on est toujours le moderne ou le post-moderne de quelqu'un), voire cyclique (on retombe dans le renouvellement cyclique des antiqui et des modermi au Moyen Âge). Le relativisme historiste et l'éclectisme vident les moments empruntés au passé de ce que Baudrillard appelle leur substance, alors que la mode comme identification provisoire de l'actuel, de l'instant de décision, est à la recherche d'une substance : en réactualisant tel ou tel fragment du passé, elle se donne la possibilité de s'opposer comme moment dialectique à l'homogénéisation des contenus dans la continuité de la temporalité d'entendement. Ce moment qu'elle oppose est un moment dans une dialectique substantielle ${ }^{34}$ qui recomposerait les moments dissociés. On voit au demeurant au passage comment le post-modernisme, sous couvert de pluralité et d'éclectisme, obéit en fait à la logique de l'homogénéisation puisque tous les moments, tous les emprunts, tous les jeux de discours ont la même valeur, sont absolument équivalents, dépouillés de leur singularité au moment même où celleci semble reconnue. Anything goes ${ }^{35}$.

32. BAUDRILLARD, art. "Modernité ", in Encyclopadia Universalis, p. 140.

33. LYOTARD, "Réponse à la question : qu'est-ce que le post-moderne? ", op. cit.

34. HABERMAS, "La modernité . . . ", p. 957.

35. B. SCHMIDT, "L'ail c'est bon, le chocolat, c'est bien : l'ail et le chocolat doivent être bon ensemble ", in Catalogue du Festival d'Automne de Paris, 1982. 
Dans les modèles dialectiques d'Adorno et de Benjamin, l'illumination profane et la constellation inaugurent en revanche une nouvelle forme d'expérience; s'identifiant à une singularité, elle se nomme chez Benjamin expérience auratique et c'est à cette même notion d'aura qu'Adorno et Horkheimer recouraient en 1944 dans la Dialectique de la Raison pour désigner le sauvetage du singulier dans un mode de connaissance pour lequel « l'art est le modèle de la science ${ }^{36}$. Nous avons montré plus haut en quel sens la constellation méritait la dénomination d'expérience esthétique. Cette expérience esthétique n'a cependant de sens que dans le mouvement d'auto-réflexion du système ; nous avons vu que la philosophie " remâche " en quelque sorte l'échec de la modernité et, en " faisant entendre " l'impuissance, répète en somme l'expérience de l'art moderne. Mais l'auto-réflexion construit la rupture, lui assigne un statut dialectique et fait qu'elle ne se replie pas «sur la posture accusatrice de l'œuvre d'art ésotérique " ainsi que Habermas le dit de la Théorie esthétique d'Adorno ${ }^{37}$. Repliée sur elle-même, la modernité esthétique n'est que "l'ombre de la révolution manquée, sa parodie ${ }^{38}$. Rien de plus funeste donc qu'une expérience esthétique qui viendrait prendre la relève de l'impuissance de la Raison. C'est alors qu'elle succomberait à la contradiction qu'on vient de développer et qui fait que la saisie du singulier comme singulier, sans aucun moyen d'en assimiler la substance, sombre paradoxalement dans l'indistinction, l'homogénéisation. L'expérience esthétique du singulier, du décomposé, soit l'expérience de la rupture, doit être réinscrite dans une auto-réflexion dialectique. Elle se condamne sinon au destin de l'avant-garde moderne : concevoir et tenter de transformer l'histoire à partir de l'esthétique - projet voué à l'échec. Dans «Échec de la Nouvelle Gauche », Marcuse renouvelle sur le terrain politique ce constat d'échec formulé par Adorno à propos de la modernité esthétique. En revanche, l'approche esthétique s'inscrit logiquement dans une conception dialectique de l'histoire pour laquelle la reconstruction de la totalité d'un mode de production consiste précisément à y déceler les germes de sa décomposition et n'est possible qu'au moment où cette décomposition est entrée dans une

36. Dialectique de la Ratson, p. 36.

37. "La modernité ... ", p. 968.

38. LEFEBVRE, op. cit., p. 174 ; vorr sa remarque sur la terreur. 
phase décisive. C'est d'une telle phase décisive que rendait compte la bi-polarisation sociale chez Marx. Or, quand bien même cette bi-polarisation ne serait plus à l'ordre du jour, rien n'empêche que l'on tente de saisir la décomposition comme un phénomène dialectique. Si on ne peut plus s'appuyer aujourd'hui sur la totalité lukacsienne, que n'incarne plus aucun représentant de l'universel concret (le « point de vue du prolétariat " s'opposant à l'expérience bourgeoise de la scission) ${ }^{39}$, la saisie du positif sur le versant de sa négativité (Marx, Postface de 1873 à la seconde édition allemande du (apital) reste possible. Bloch souligne dans Le Principe Espérance que ce qui est important dans une totalité - qu'il s'agisse de la belle totalité de l'œuvre d'art classique ou de la totalité d'un mode de production - c'est le mouvement de sa décomposition ${ }^{40}$; de cette décomposition naît le carpe diem révolutionnaire - idée très proche de l'instant messianique de Benjamin. D'où l'intérêt d'une "investigation des phénomènes symptomatiques de l'époque ${ }^{41}$; lorsqu'il s'agit d'une enquête sur le singulier comme décomposition d'une totalité, c'est-à-dire lorsqu'on ne s'installe pas dans la rupture en renonçant a priori à la notion de totalité ; à titre de recensement des déficits d'une fausse totalité, des atteintes subies par les mécanismes de là socialisation et par les motivations dans l'accomplissement d'un certain type de rationalité, il y a sans doute beaucoup à retenir du diagnostic deleuzien de la déterritorialisation ou bien encore du projet de « microphysique du pouvoir » de Foucault.

Dans les formes très tenues et très localisées d'une éthique communicationnelle recomposant ce qui fut dissociét2 ${ }^{2}$, on ne peut certes voir rien de plus que des constellations provisoires, synthèses révocables d'universel et de particulier, utopies localisées, concrétisations «esthétiques» d'une rationalité non réduite. La vie nouvelle ${ }^{43}$ qui s'esquisse en elles n'est pas représentable. Elle est sujette à cette interdiction juive de la repré-

39. LUKACS, Histoire et conscience de classe.

40. BLOCH, Das Prinzip Hoffnung, 1959, traduction française de F. WULlmarT, Gallimard, 1976 : chap. 20.

41. Habermas, "Stichworte zur geistigen Situation der Zeit ", Suhrkamp, 1979.

42. Raison et légitimité, p. 126 et Stichworte, op. cit., traduction française, p. 51.

43. J'utilise cette expression parce qu'elle eut son heure de gloire en esthétique ; voir l'essai de Benjamin sur Proust. 
sentation qui caractérise l'utopie adornienne ${ }^{44}$. Celle-ci relève bien plutôt du sublime. Leur avantage sur la pure révolte esthétique habitée elle aussi de sublime (cf. supra Kleist) est que sous la forme certes subjective du Beau elles témoignent de la possibilité d'une recomposition.

Le sublime sans la constellation, c'est l'échec dans la terreur. Pour conclure ce paragraphe, je voudrais rapidement développer cette idée qui, à mon sens, indique l'impasse de la post-modernitésublime que Lyotard oppose à la terreur. Cette définition traduit clairement le refus de toute identification, fût-elle provisoire et révocable, et de toute recomposition dialectique. Le sublime était, déjà chez Kant et plus encore chez Schiller, ce qui interdit au système de se clore et au Beau d'être une synthèse définitive. La démarche de Schiller est l'effort désespéré pour rendre le sublime commensurable à l'histoire. Pourquoi ? Parce que le sublime et la terreur vont de conserve ; Hegel l'a montré à propos de la révolution française. L'impossibilité de nommer le sublime, c'est la possibilité de la terreur toujours ouverte. On s'étonnera donc de voir Lyotard - au prix de raccourcis stupéfiants opposer une esthétique du sublime (post-modernisme qui " invente des allusions au concevable qui ne peut être présenté $")^{45}$ à la terreur. Pur wishful thinking, à nouveau, reposant sur l'espoir déjà souligné que le post-moderne inaugurerait " en principe »- une démocratie incomparable. Selon Lyotard la terreur naîtrait de l'illusion transcendantale hégélienne qui, confondant la pensée et le réel, totalise des jeux de langage distincts (les trois facultés kantiennes). Il s'agit là d'une assimilation abusive du totalitarisme à la terreur. Ce qui s'accomplit chez Hegel, c'est le totalitarisme. Celui de la Raison. Rendant toutes choses commensurables, ce totalitarisme est celui d'une identification de la Raison à l'entendement et d'un jeu de langage, comme le montre la Dialectique de la Raison. C'est parce qu'il s'agit d'un jeu de langage particulier, celui du concept, que la terreur devient son instrument. Mais, pour cette raison même, la terreur existe avant le totalitarisme : comme Raison chez Robespierre, comme la tyrannie de la morale dénoncée par

44. ADORNO, Théorie esthétique, traduction française de M. JIMENEZ, Klincksieck, 1974 : p. 50sq.

45. Lyotard, "Qu'est-ce que le post-moderne ? ", Critique, avril 1982 : p. 367. 
Schiller. Et cette terreur là est indissociable du sublime. S'il n'est pas douteux que le désir de faire la paix avec le réel est inspiré par le totalitarisme et l'alimente, le sublime lui engendre la terreur. Dans l'option post-moderne de Lyotard, la terreur est celle d'un esthétisme coexistant avec l'aspiration totalitaire du jeu de langage stratégique sans chances réelles de mettre fin à ce dernier si elle n'entre pas dans une dialectique avec la rationalité instrumentale. D'où l'alternative insoluble qui conclut $L a$ condition post-moderne. La coexistence d'une gestion aux aspirations totalitaires et du terrorisme n'est que trop réelle. Contre l'esthétique du Beau et la mélancolie de la modernité obsédée par la perte du sens (" traurige Wissenschaft " chez Adorno, allégorie chez Benjamin), Lyotard voit dans un post-modernisme "alléguant l'imprésentable chez la présentation " ${ }^{46}$ comme la perspective d'un Gai Savoir. Perspective tragique qui surmonterait le nihilisme, au sens nietzschéen de ce terme. Or, l'opposant à la fois au moderne et à la "post-avant-garde ", il la définit seulement par la remise en cause perpétuelle des moyens de l'art ${ }^{47}$. Faute donc de pouvoir être autre chose que ce que fut déjà la modernité ${ }^{48}$, le post-modernisme conçu ainsi s'offre au destin du modernisme de Weimar : l'impuissance face au totalitarisme, scellée par une continuelle rupture qui se consume dans le geste qu'elle consomme.

\section{RECOMPOSER LES JEUX DE DISCOURS}

Coupé des autres actes du langage, le jeu de discours de l'esthétique s'enfonce ici dans l'autisme, figure extrême de l'autonomie dans le contexte de ce que Deleuze appelle le capitalisme schizophrène ${ }^{49}$. L'autonomie et l'autarcie font symptomatiquement partie des fantasmes protestataires du capitalisme avancés ${ }^{50}$. Ils représentent un repli défensif dans la particularité ${ }^{51}$, à l'exception du mouvement d'émancipation des femmes, qui montre

46. Ibid., p. 364 .

47. "Travail vertigineux des questions lancées aux règles de l'image et du récit . . ., accélération, les 'générations> se précipiteront ", ibid., p. 365.

48. "Le post-moderne fait assurément partie du moderne ", ibid.

49. Capitalisme et schizophrénie, op. cit.

50. RAULET, "Consensus et légitimité ", in Esprit, janvier 1980 : p. 169 - sur une " dialectique de la schizophrénie » à partir d'Adorno, ibid., p. 168sq, et « Retour du religieux et rationalité technocratique ", in Lumière et vie, $\mathrm{n}^{\circ} 148$.

51. HABERMAS, TKH, p. 576sq. 
seul un caractère offensif et peut être encore conçu dans l'optique des mouvements de libération bourgeois et socialiste ${ }^{52}$, mais se caractérise lui aussi par son particularisme. Les réactions suscitées par les atteintes aux «fondements organiques du monde vécu » (destruction de l'environnement, pollution, etc.), les peurs suscitées par une nouvelle catégorie de risques à long terme et qui trahissent en fait l'incapacité de nos facultés de compréhension face à l'hypercomplexité du système ou encore le repli sur des communautés communicationnelles (provincialisme, sectes, etc.) permettant une identité personnelle et collective sont des effets de la modernisation ${ }^{53}$; à ce titre elles ne justifient nullement la simple acceptation de la juxtaposition mais requièrent qu'on en reconstruise la logique. Par cette reconstruction, ces ruptures trouvent leur place dans une dialectique historique à laquelle il n'y a pas lieu de renoncer. Ce qui ne signifie certes nullement qu'elles puissent être recomposées, totalisées, organisées, polarisées comme pouvait le faire le marxisme traditionnel en reconstruisant la totalité du mode de production capitaliste, en dégageant une bi-polarisation des conflits et en organisant dans cette optique les diverses révoltes autour d'une contradiction principale (cf. Manifeste du Parti communiste). Habermas signale les parentés qui existent entre les protestations actuelles et des révoltes non-contemporaines du siècle dernier ${ }^{54}$. L'énorme difficulté à laquelle se heurte la conception orthodoxe de l'organisation est l'impossibilité de déterminer le pôle qui les unifierait, "le maillon le plus fort ${ }^{55}$. En désignant certaines protestations comme "néo-populistes ", Habermas fait allusion à la notion de «peuple » utilisée par Marcuse à partir de Contre-révolution et révolte : l'opposition du capital et du travail se serait, selon Marcuse, exacerbée en une opposition du capital à l'ensemble de la population. On peut encore expliquer autrement les nouveaux conflits et y voir une " colonisation " croissante du mode vécu par les impératifs du système, une destruction des formes traditionnelles d'intégration sociale et notamment une atteinte aux structures communicationnelles

\footnotetext{
52. Il lutte comme eux contre une forme de vie patriarcale, Ibid., p. 578.

53. HABERMAS, TKH, p. 580sq.

54. HABERMAS, TKH, p. 577sq.

55. Marcuse, cf. RAULET, "La révolution impossible ? En hommage à Herbert Marcuse ", in Allemagnes d'Aujourd'bui, septembre-octobre 1979.
} 
(Habermas $^{56}$. Quoi qu'il en soit, le problème de l'organisation reste entier. L'universalisation de la contradiction et la tendance à une colonisation totale se traduisent par un morcellement généralisé. La décomposition impose une logique nouvelle qui privilégie le particulier sur la totalité ${ }^{57}$. L'éclatement du monde vécu en sphères autonomes, soit la logique même qui avait engendré la modernité culturelle ${ }^{58}$, s'est différencié encore ${ }^{59}$. On peut, avec Marcuse, proposer des "stratégies minimales de front unique ", des "bases locales et régionales" tentant d' " articuler la protestation ${ }^{60}$ et dans lesquelles je suis tenté de voir des constellations ; mais pour cette raison même, la recomposition qu'elles opèrent demeure particulière. La problématique actuelle de l'émancipation doit en tous cas prendre en compte une rupture radicale avec le modèle traditionnel, et peut-être même avec l'idée de l'accomplissement de la raison dans l'histoire, puisqu'aussi bien elle s'est accomplie comme décomposition ${ }^{61}$.

\section{CONCLUSION}

La question de l'émancipation, on l'aura compris, reste entière. Elle dépend dans les faits de l'aptitude d'agents sociaux, plus exactement de constellations consensuelles, à prendre en charge la rationalité non-réduite et à recomposer ce qui fut dissocié. Mais on a au moins dégagé les conditions de possibilité d'un nouveau réalisme - un réalisme dialectique puisqu'il remettrait en branle, en quelque sorte en sens inverse, depuis son enlisement dans la décomposition, la dialectique de la modernité ou, si l'on veut formuler autrement cette idée, réintroduirait une dialectique dans la clôture de la Raison. Idée essentielle, même si elle est paradoxale, car c'est son paradoxe qui permet la reprise : la continuité fut scission, l'enferment décomposition. La post-modernité n'est pas nécessairement l'impasse d'une décomposition abolissant la Raison et dont seule

56. Cf. Stichworte, op. cit., et Theorie des kommunikativen Handelns.

57. Sur ce renversement de perspective, cf. RAULET, " La révolution impossible . . . ", op. cit., p. 38sq.

58. HABERMAS, TKH, p. 564 et " La modernité . . . ", p. 958sq.

59. Stichworte, traduction française, op. cit., p. 45 sq.

60. Marcuse, cf. RAULET, « La révolution impossible . . . ", op. cit.

61. Sur la nécessité de cet abandon, cf. HABERMAS, TKH, p. 561sq. 
pourrait nous faire évader une Grande Rupture qui ressemble un peu trop à l'amor fati d'un nouveau positivisme - au mieux à la Bejabung nietzschéenne. La rupture peut être dialectisée comme l'instant d'une décision dans le destin de la rationalité. S'il faut renoncer au modèle d'émancipation de la Raison dans l'Histoire, on peut ne renoncer ni à la Raison, ni à l'Histoire, ni à la dialectique. Le nouveau réalisme implique que leurs liens soient redécouverts dans un processus qui n'est ni homogène... et doit miser pour cette raison même sur des constellations ... le particulier. À cette condition, la post-modernité pourrait être une modernité renouvelée, non au sens d'une répétition et d'une aggravation de l'échec moderne mais d'une relance. À cette même condition, la partialité pour la Raison peut n'être pas seulement un pur idéal ou un parti-pris polémique conduisant à s'installer dans le rationalisme comme on s'installe dans l'irrationalisme.

Institut d'études germaniques Université de Paris IV 